\title{
The Plenhaptic Guidance Function for Intuitive Navigation in Extended Range Telepresence Scenarios
}

\author{
Antonia Pérez Arias, Henning P. Eberhardt’ Florian Pfaff; and Uwe D. Hanebeck ${ }^{\S}$ \\ Intelligent Sensor-Actuator-Systems Laboratory (ISAS) \\ Institute for Anthropomatics \\ Karlsruhe Institute of Technology (KIT) \\ Karlsruhe, Germany
}

\begin{abstract}
In this work, we propose a plenhaptic guidance function that systematically describes the haptic information for guiding the user in the target environment. The plenhaptic guidance function defines the strength of the guidance at any position in space, at any direction, and at any time, and takes the geometry of the target environment as well as all possible goals into account. The plenhaptic guidance function, which can be rendered as active and passive guidance, is sampled and displayed to the user through a haptic interface in the user environment. The benefits of the plenhaptic guidance function for guiding the user to several simultaneous goals while avoiding the obstacles in a large target environment are demonstrated in real experiments.
\end{abstract}

\section{INTRODUCTION}

Extended Range Telepresence aims at giving a human user a realistic impression of being present in either a real remote environment or a virtual environment. The feeling of presence is achieved by visual, acoustic, and haptic information, which is recorded in the target environment and then presented to the user on an immersive display. At the same time, the user motion is tracked and transferred to the proxy in the target environment. Thus, the degree of immersion of the user is increased [17] and the navigation in the target environment becomes much more intuitive. However, without further processing of the motion information, the motion of the human operator is restricted to the size of the user environment. Motion Compression [12] solves this problem by mapping the desired path in the target environment to a feasible path in the user environment by minimizing proprioceptive and visual inconsistencies.

Many telepresent tasks require a high-precision motion and/or a high speed, which makes them difficult to perform and to learn [18]. Preliminary studies have demonstrated that haptic guidance approaches can improve precision and speed as well as reduce training time in such tasks [14]. This can be achieved through virtual constraints, called virtual fixtures, that redirect undesirable operator motions towards useful directions. With virtual fixtures, speed, accuracy, and safety are greatly enhanced in many tasks [13]. Much work has been done in virtual fixtures $[1,5,11,18]$. However, a general problem of virtual fixtures is the lack of flexibility of the guidance functions, for example, when the user is only able to walk towards a target position or along a certain path.

Some research work tries to adapt the guidance functions to the user intention $[6,7,10]$, so that only the intended motions of the user are assisted. However, the resulting guidance functions are either very simple, as in [6], where the user is assisted in accelerating

\footnotetext{
*e-mail: antonia.perez@kit.edu

†e-mail: henning.eberhardt@kit.edu

†e-mail: florian.pfaff@student.kit.edu

§e-mail: uwe.hanebeck@ieee.org
}

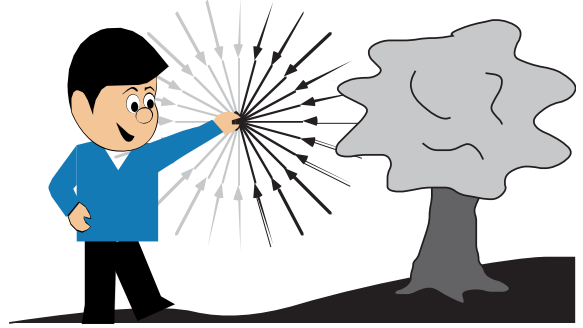

Figure 1: The plenhaptic guidance function (PHGF) describes the haptic guidance information available to a user at any point in space and time. The analogy with the plenoptic function [2] is shown here. The user is in the center of all guidance directions. The gray value of the rays corresponds with the intensity of the guidance at the direction of the arrows.

and decelerating motions, or depend on exact task information, e.g., in $[7,10]$, where the guidance in a modified environment or towards an alternative goal would require the laborious task of generating and training new models.

Potential field methods have been largely used for path planning of autonomous robots and obstacle avoidance [3, 8, 19]. The main advantage of potential field methods is that they are easy to compute and can manage context information by superimposing several geometric shapes [3]. The user could be guided in the target environment by using repulsive force fields around the obstacles to be avoided and attractive force fields around target regions. However, by using such force fields, the user would permanently perceive a force in the direction of the force field. Thus, the degree of user control would be quite restricted and the user would be constrained to walk towards the direction dictated by the force field.

In this paper, we propose a new systematic approach to design a haptic guidance function that simultaneously considers both contextual information from the environment and several potential goal locations, so that the user can freely choose between one of these goal positions. This haptic guidance function is called plenhaptic guidance function (Fig. 1).

The remainder of this paper is structured as follows. In Sec. 2, the problem of haptically guiding a user in a large-scale environment is defined. Sec. 3 presents the idea of the plenhaptic guidance function and Sec. 4 discusses the new systematic approach to calculate this guidance function. Sec. 5 presents two methods to render the haptic information, while Sec. 6 provides some simulation results. Real-world experiments are presented in Sec. 7 and the paper is concluded in Sec. 8 .

\section{Problem Formulation}

We assume a bilateral telepresence system, in which the head position of the operator is sent to the proxy that replicates his motion. Interaction forces as well as visual and acoustic information from the target environment are sent back to the operator and displayed 
on a haptic interface and a head-mounted display with headphones, respectively. In our system, the operator uses his own motion, i.e., natural walking, to navigate the proxy. Wide-area motion in arbitrarily large target environment is possible by employing Motion Compression [12], which maps the position of the user between the limited user environment into the target environment.

In many applications of telepresence that demand high accuracy or high speed, it is convenient to assist the human operator in navigating the proxy for carrying out a structured task in a large target environment. Therefore, in previous work [16], we proposed a wide-area haptic guidance method that augments the haptic information from the target environment with force cues and, in combination Motion Compression, allows to guide the operator on arbitrarily large target paths. However, the systematic consideration of context information, an easy application to different scenarios, and the trade-off between user guidance and operator control are still difficult challenges in the design of haptic guidance functions.

Given information about the geometry and the points of interest of a scene in the target environment, a guidance function is sought that assists the user in reaching the possible goals but still leaves the operator some control to choose and change his goal. This function has to fulfill the following requirements:

1. at each location there is at least one direction that leads towards the goal,

2. the user is able to choose his goal when several simultaneous goals exist, and

3 . the motion of the user is only completely restricted when avoidance of close obstacles is required.

\section{Plenhaptic Guidance Function}

A human operator can learn about three-dimensional objects in the scene by means of active exploration through touch. By exploiting the analogy with the plenoptic function [2], we define the plenhaptic function that describes the intensity of the haptic information in the scene. The plenhaptic function specifies formally the amount of haptic stimuli passing through the human's hand, and/or the human's fingers for a given position in the space, a given exploration angle, and a given time. The task of the haptic perception is to extract a useful description of the plenhaptic function's properties in terms of, for example, experienced forces. The actual information extracted by the human haptic perception system is a small subset of the information that is physically available in the plenhaptic function, since humans gather haptic information from a certain number of positions at a time, obtaining samples from each exploring direction.

In the same way, when guiding a human operator in the target environment, the additional haptic information that is used to guide the human can be described by a function that will be called the plenhaptic guidance function (PHGF). In fact, the plenhaptic function describes the total amount of haptic information, i.e., not only the haptic information provided by the objects in the scene but also the haptic guidance information.

As in this work we are mainly interested in the haptic guidance information, we will concentrate on the $P H G F$. This $P H G F$ is a continuous function that describes the intensity of the haptic guidance at any location $\underline{x}$, at any possible exploring direction defined by the angle $\alpha$, and at any time $t$. The resulting function takes the form

$$
P H G F=P H G F(\underline{x}, \alpha, t) .
$$

Fig. 1 illustrates the analogy between the plenhaptic guidance function and the plenoptic function. Actually, the intensity of the guidance along a certain direction towards an obstacle will be higher than towards another direction without obstacles.

\section{Calculation of the PHgF}

Our goal is to find a guidance function, the $P H G F$, that completely defines the haptic information required for haptically guiding the user in a large and arbitrarily structured target environment, while simultaneously considering environmental constraints and several goal locations. However, this function is not unique. In this section, a particular $P H G F$ is calculated that systematically considers the context information of the scene and provides for an adequate haptic guidance, which fulfills the given requirements.

\subsection{Potential Function}

To calculate the $P H G F$, we first define a potential function for each possible goal that takes all obstacles into account. The potential function, which penalizes the vicinity of the obstacles and rewards the proximity to the goal, consists of three different components: the goal, the geometry of the target environment, and the geometry of the user environment.

Taking only the geometry of the target environment into account can result in guidance that, while leading the user to goals in the target environment, leads the user past the boundaries of the user environment. To solve this problem, the boundaries of the user environment can be transformed into the target environment and handled as moving obstacles at each time instant.

The potential function, which considers all the obstacles and one possible goal $i$ and depends on the location $\underline{x}$ and the time $t$, shall be called $P_{O G_{i}}(\underline{x}, t)$. The potential function is strictly monotonically decreasing towards the goal location, which is the global minimum of the function. This is necessary in order to guarantee at each location that at least one direction leads towards the goal. Diverse potential functions, e.g., harmonic functions, can be used. The use of a potential function for each possible goal is required to allow possible simultaneous goals.

\subsection{Calculation of PHGF for one Goal}

A user motion model is taken into account to derive the $P H G F$ from the potential function. The motion model assumes that the user walks at each time step a distance $s$ in the direction described by the angle $\alpha$. If the potential function at the next position has a lower value than at the current position, the user will approach to the goal, whereas if the potential function has a higher value, the user will approach to an obstacle by walking in the direction described by $\alpha$.

The value of the plenhaptic guidance function for a certain goal $i, P H G F_{i}$, can then be calculated by means of the directional derivative $D_{\alpha}$ of the potential function $P_{O G_{i}}(\underline{x}, t)$ defined by

$$
D_{\alpha}=\lim _{s \rightarrow 0} \frac{P_{O G_{i}}(\underline{x}+s \underline{u}(\alpha), t)-P_{O G_{i}}(\underline{x}, t)}{s},
$$

as follows

$$
P H G F_{i}(\underline{x}, \alpha, t)=\operatorname{norm}\left(D_{\alpha}\right),
$$

where $\underline{u}(\alpha)$ is a unit vector in the direction $\alpha$, and norm $(\cdot)$ is a normalization function that is explained in the following. Fig. 2 illustrates how the function $P H G F_{i}$ is derived from a potential function.

We assume from here on that the $P H G F_{i}$ is normalized, i.e., returns positive values between zero and one. The $P H G F_{i}$ will return zero in the direction towards the goal, while the value one corresponds to a direction that has to be avoided by all means because it leads to a close obstacle.

The normalization function consists of a local offset and a global scaling. First, the normalization function adds an offset to the directional derivative so that the $P H G F_{i}$ is always positive and the minimum value of the $P H G F_{i}$ at each location is set to zero. By 


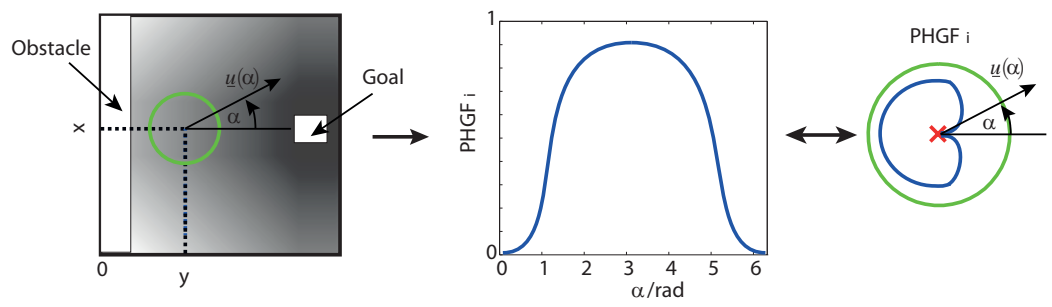

Figure 2: The $P H G F_{i}$ at a certain location $(x, y)$ can be calculated from the potential function (left) as the normalized directional derivative in the direction defined by $\alpha$. The $P H G F_{i}$, which returns positive values between zero and one, is represented in polar coordinates (right).

doing so, there is always at least one direction at each location in which the user should walk. Second, the maximum value of the $P H G F_{i}$ in the whole scene is set to one, so that at any other location all values of the $P H G F_{i}$ are less than or equal to one. By doing so, the obstacles are strongly penalized just in its immediate vicinity and the motion of the user is only restricted when avoidance of close obstacles is required.

\subsection{Calculation of PHGF for several Goals}

In the previous subsection, the plenhaptic guidance function $P H G F_{i}$ for each goal $i$, was obtained. However, in a large target environment several goals can exist. These goals might be simultaneous, in which case the user may decide which goal he wants to walk to, or exclusive, if the user has to walk towards the nearest one.

In order to allow the user to reach simultaneous goals, and assuming the resulting $P H G F$ is zero along the possible guidance directions, the functions $P H G F_{i}$ for each goal have to be combined with a certain t-norm [9]. The combination of the functions $P H G F_{i}$ with a t-norm makes it possible to walk to simultaneous goals because the zeros of the $P H G F_{i}$ are preserved in the resulting $P H G F$ at the same locations. Furthermore, since the $P H G F_{i}$ are derived from potential functions that consider the same obstacles, all $P H G F_{i}$ will have large values in the directions towards the obstacles and the information of the resulting $P H G F$ will be consistent. The $P H G F$ is obtained as follows

$$
\operatorname{PHGF}(\underline{x}, \alpha, t)=\underset{i=1}{G}\left\{\operatorname{PHGF}_{i}(\underline{x}, \alpha, t)\right\},
$$

where $G$ is the number of simultaneous goals. Fig. 3 shows an exemplary $P H G F$ for two simultaneous goals at the directions $\alpha=$ 0 and $\alpha=\pi$. By combining the functions $P H G F_{i}$ for each goal with the minimum norm, the resulting $P H G F$ allows the user to choose between the two potential goal locations. Since the minimum norm is the largest t-norm [9], by using other t-norm the level of guidance towards the non-preferred directions will tend to be lower.

On the other hand, in order to guide the user to exclusive goals, a potential function $P_{O G}$ for all exclusive goals has to be calculated. The $P H G F$ is then derived from this potential function, which results from the conjunction of the individual potential functions $P_{O G_{i}}$ for each exclusive goal.

A time-variant $P H G F$ is necessary when dealing with moving obstacles or dynamic scenes. As the potential function has to be calculated globally, its calculation is largely dependent on the area of the scene $A$. Due to the necessity to calculate the $P H G F_{i}$ for all goals $G$, the resulting complexity for the potential function calculation becomes $\mathscr{O}(A G)$. The derivative calculation for all discrete directions $\mathrm{N}$ at the current point $\underline{x}$ adds further, but usually negligible, complexity of $\mathscr{O}(N)$. In the case that the number of goals increases drastically, the computation time can be reduced by reducing the spacial resolution of the $P H G F$.

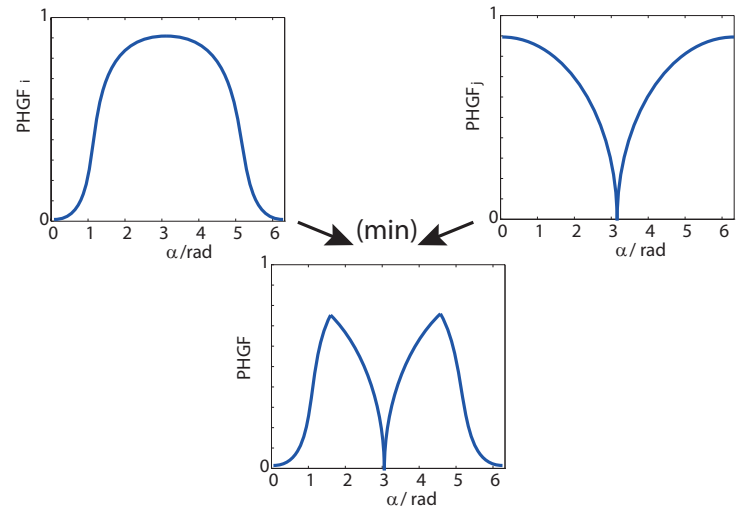

Figure 3: The $P H G F$ for two simultaneous goals at the directions $\alpha=0$ and $\alpha=\pi$ is obtained by combining the individual functions for each goal $P H G F_{i}$ with the minimum t-norm.

\section{Rendering of PHGF}

Once the $P H G F$ is calculated, we can render the guidance information in different ways. As described in [16], there are two possibilities to render the haptic guidance information with a haptic display by using active and/or passive guidance.

\subsection{Active Guidance}

When using active guidance, the user is guided towards his goal by means of an additional guidance force that pulls him towards the desired direction.

The guidance force takes the general form

$$
\underline{F}_{G}=\underline{F}_{G}(\underline{x}, \alpha, t)
$$

Because the guidance force at each instant is directed towards the desired direction, the only possibility to guide a user towards a goal with active guidance is to render the $P H G F$ as a certain positive guidance force just along the direction $\alpha$ in which the $P H G F$ has a zero value and zero guidance force in the other directions. Please note that this rendering would provide a similar guidance to that of the force field methods.

However, when several simultaneous goals exist, i.e., the $P H G F$ has zero values at several directions, a rendering is required that still allows the user to choose his goal. One possibility to do this is to detect which of these goal directions the user wants to walk to, for example by measuring the applied force, and assist the motion in this direction with a positive guidance force.

In contrast to the active rendering of the $P H G F$, the passive rendering of the $P H G F$ allows the display of a continuous guidance in all directions. 


\subsection{Passive Guidance}

With passive guidance, the user is guided by attenuating the motion in the different directions. The velocity of the manipulator can then be written in absence of contact force from the target environment as

$$
\underline{v}_{G}(\underline{x}, \alpha, t)=C(\underline{x}, \alpha, t) \underline{F},
$$

where $\underline{v}_{G}$ is the commanded velocity of the manipulator, $\underline{F}$ is the force applied by the user, and $C(x, \alpha, t)$ is an anisotropic admittance matrix that attenuates the motion of the end-effector along the direction $\alpha$ of the applied force at the user location $x$ and instant $t$.

Thus, the passive rendering of the PHGF simulates a set of mass-spring-damper elements in all directions at a given position, so that when the user moves, for example, towards an obstacle, his motion is accordingly damped. The value of the damping at each direction is obtained by mapping the values of the $P H G F$ between the minimum and the maximum damping that can be displayed by the haptic device with a monotonically increasing function.

\subsection{Control of Haptic Display}

The haptic information is presented to the user on a haptic display. In our system, the haptic interface is modelled as an admittance, which transforms the reference force to be displayed into the reference motion of the end-effector. The admittance model, which shapes the desired dynamic behaviour of the system by compensating the natural device dynamics, allows the display of arbitrary forces at any position and also to display the different admittances (mass, dampers, and springs) in any direction.

The control law of the haptic display becomes

$$
\underline{v}_{r e f}(\underline{x}, \alpha, t)=C(\underline{x}, \alpha, t)\left(\underline{F}-\underline{F}_{r e s}\right),
$$

where $\underline{v}_{r e f}$ is the reference velocity of the manipulator, and $\underline{F}_{r e s}$ is the total force presented to the user that results from

$$
\underline{F}_{r e s}=\underline{F}_{G}+\underline{F}_{T},
$$

where $\underline{F}_{G}$ is the active guidance force and $\underline{F}_{T}$ is the contact force from the target environment. Thus, the force control of the display allows the combination of the active and the passive rendering of the $P H G F$.

\section{Simulation Results}

For evaluating the proposed approach, a typical building scenario, displayed in Fig. 4, was considered. In this scenario there are several possible goals. One of them is the conference room (the largest room on the right side of the building) and the other possible goals are the exits of the building. In order to illustrate how the PHGF works, we assume that the user may either go to the conference room or leave the building. Although three exits exist, only the closest one to the user is of interest for him. Therefore, the three exits are exclusive goals, and the conference room and the closest exit are simultaneous goals.

The desired $P H G F$ is calculated as follows: First, the potential function for the obstacles is calculated. Fig. 4 shows the potential function of the obstacles (top). A square function is used here to penalize the proximity to the obstacles. For clarity, merely the obstacles in the target environment were considered here. The potential function of the obstacles and the first goal (conference room) is calculated (Fig. 4 (left)) using a potential function free of local minima. At the same time, the potential function for all three exits is generated in a similar way (Fig. 4 (right)). Please note that by calculating only one potential function for all three exits, the gradient of the potential function will always point to the closest exit. Finally, a $P H G F_{i}$ for each goal is derived from each potential function.

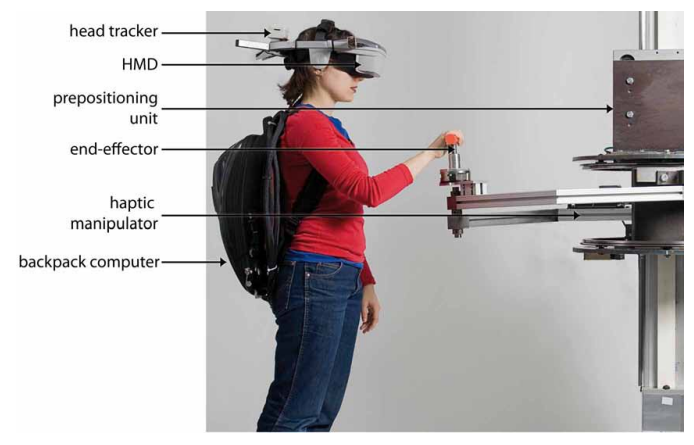

Figure 5: User interface in our telepresence system. The position of the user is tracked and sent to the target environment. This is used to sample the PHGF. The user sees the target environment through a head-mounted-display and the haptic manipulator displays the haptic information from the PHGF.

In order to allow the user to choose between different goals (conference room and closest exit), both functions are merged by the minimum norm. Fig. 4 (bottom) shows the resulting PHGF at several locations on a grid and for all directions and Fig. 4 (middle) shows an upscaled view of the $P H G F$ at one location. The green circle represents all possible directions while the radius of the blue line represents the value of the $P H G F$ in a specific direction. The simulation results show that the $P H G F$ is well capable of guiding the user either to simultaneous or to exclusive goals while avoiding the obstacles.

\section{Real Experiments}

\subsection{Setup}

Real experiments were conducted using a large haptic interface specifically designed for extended range telepresence [15]. Fig. 5 shows the user interface used for wide-area haptic guidance. The haptic interface consists of two subsystems: a linear prepositioning unit that moves with the user along the user path and a manipulator arm attached to the prepositioning unit that is used to display defined forces at any position in the user environment. In this way, the workspace of the haptic interface covers the whole user environment. The prepositioning unit is realized as grounded portal carrier system of approximately $5 \times 5 \mathrm{~m}^{2}$. A typical motion speed of $2 \mathrm{~m} / \mathrm{s}$ and an acceleration of $2 \mathrm{~m} / \mathrm{s}^{2}$ are achieved. The manipulator arm covers the human arm workspace and allows for planar movement of the end-effector.

\subsection{Scenario}

The benefits of the PHGF to guide the user to simultaneous goals is evaluated in a real experiment, in which the user has to reach a sequence of goals in a virtual building. The building plan, which is $4 \times 4 \mathrm{~m}^{2}$ large, is shown in (Fig. 6(a)). There are three simultaneous goals (G1, G2, and G3) that have to be reached twice from the start position (S) following two possible routes: S-G1-G2-G3-G2G1 (Route 1) and S-G3-G2-G1-G2-G3 (Route 2). The cumulative distance was approximately $11.5 \mathrm{~m}$ for both routes.

The $P H G F$ was generated using three harmonic potential functions, which are guaranteed to be local minimum free [4]. In addition to the Laplace's equation, Dirichlet boundary conditions along the borders of walls and targets were used. A numeric solution was obtained using the finite element method. The result of the finite element method was interpolated using Delaunay triangulation.

The PHGF is shown in Fig. 6(a) for several locations on the building plan. The $P H G F$ was rendered as passive guidance with dampers of intensity $D$ proportional to the value of the $P H G F$ in a range from $D_{\min }=10 \mathrm{Ns} / \mathrm{m}$ to $D_{\max }=400 \mathrm{Ns} / \mathrm{m}$. 


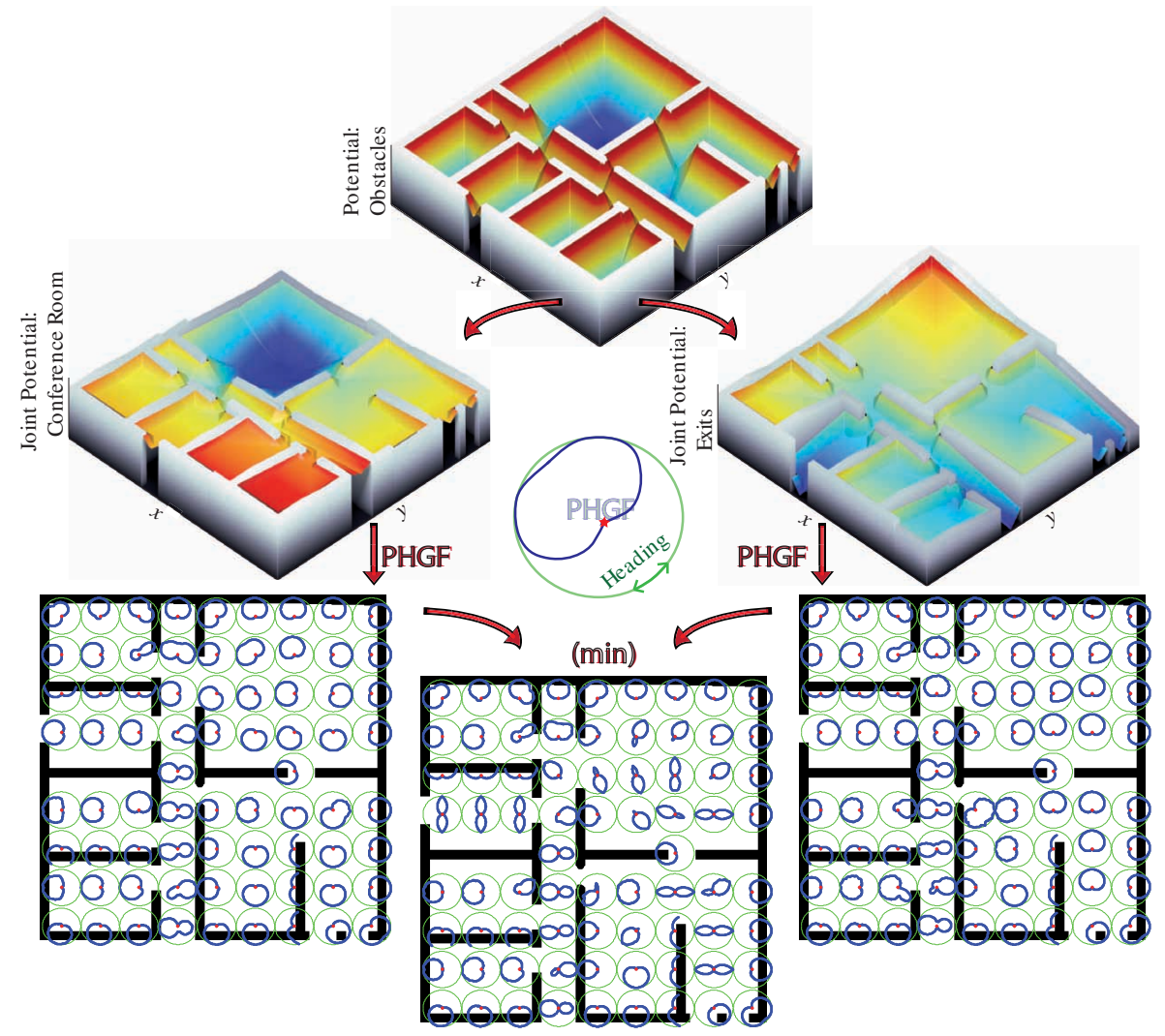

Figure 4: First, the potential function of the obstacles is generated. Then, it is joined with the potential function of the first goal (conference room) (left) and the potential function of the three exits (right). From each individual potential function a $P H G F_{i}$ is calculated. In order to allow the user to reach either the goal or the closest exit, both functions are combined with the minimum norm. In the middle, an upscaled version of the $P H G F$ is displayed.

Nine test persons were instructed to reach the goals by following each of the two routes. The execution time, the average applied force, and the distance from the obstacles were compared in order to show, first, that the user was successfully guided to the goals and second, that both routes are equivalent.

\subsection{Results}

All participants were successfully guided to the goals independently from the chosen goal sequence by avoiding collisions with virtual walls. Two sample trajectories for both routes can be seen in Fig. 6(b).

Fig. 7 shows the mean values of execution time, applied force, average distance, as well as minimum distance to the nearest wall for both goal sequences, which give an insight in the objective quality of the proposed guidance. As expected, a high resemblance of the quality criteria for both routes was achieved. This indicates, that the user can dictate at every instant the desired goal sequence, without compromising the quality of the guidance. Therefore, by using the proposed guidance, an adequate trade-off between guidance and user control is achieved.

\section{Conclusions}

In this paper, we propose a novel plenhaptic guidance function that defines the strength of the guidance at any position in space, at any direction, and at any time. This function allows for a flexible widearea guidance since it systematically considers both contextual information from the environment and several potential goal locations. Furthermore, new obstacles and potential goal locations can be easily included online.

The plenhaptic guidance function provides a general guidance framework that can be rendered both as active and passive guid- ance fixtures. Because it allows guidance to several potential goal locations simultaneously, the degree of user control is eventually increased. However, the preference for one potential goal location in the case of exclusive goals can be handled within the plenhaptic guidance function if desired.

A simple user model has been assumed in order to derive the plenhaptic guidance function from the potential functions of the goals and the obstacles. A predictive approach can be used instead that helps to choose the better path towards the targets by considering a user motion model and a time-horizon.

The usability of the plenhaptic guidance function for guiding the user to several simultaneous goals by avoiding the obstacles in the target environment has been demonstrated first in a simulation and then in a real experiment. However, a further evaluation is necessary to explore the effects of the visual guidance provided by Motion Compression on the haptic guidance and to measure the effects of the spatial resolution of the sampled plenhaptic guidance function.

The plenhaptic guidance function can be applied in a number of real applications like the training of evacuations or the navigation assistance in teleoperation tasks.

\section{REFERENCES}

[1] J. J. Abbott, P. Marayong, and A. M. Okamura. Haptic Virtual Fixtures for Robot-Assisted Manipulation. In Proceedings of the 12th International Symposium Robotics Research, pages 49-64, 2005.

[2] E. Adelson and J. Bergen. The Plenoptic Function and the Elements of Early Vision. In Computational Models of Visual Processing, pages 3-20. MIT Press, 1991.

[3] P. Aigner and B. McCarragher. Human Integration into Robot Control Utilising Potential Fields. In Proceedings of the IEEE International Conference on Robotics and Automation, pages 291-296, 1997. 


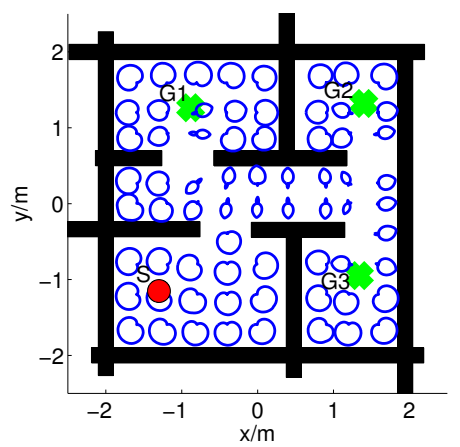

(a)

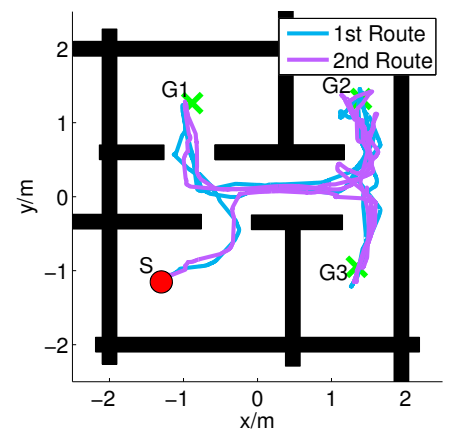

(b)

Figure 6: (a) Visualization of the $P H G F$ in polar coordinates for several locations. (b) Two exemplary trajectories of the user for two different goal sequences.

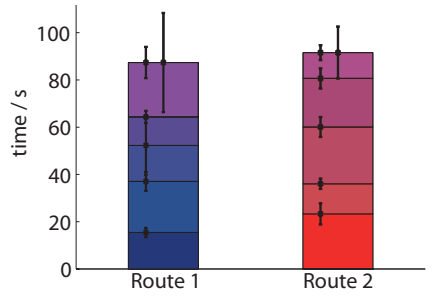

(a)

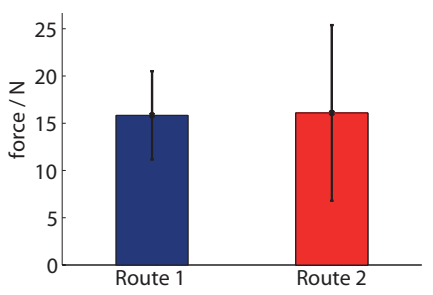

(b)

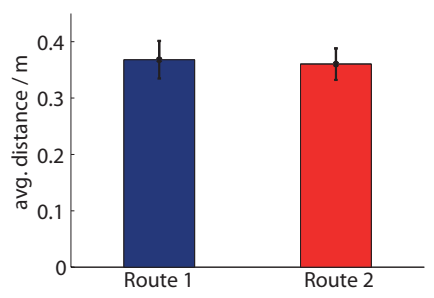

(c)

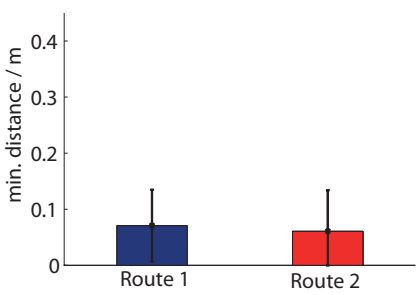

(d)

Figure 7: Average values for two different routes: (a) represents the execution time. Each segment of a bar represents the time to reach the next goal of the route. (b) shows the average applied force, (c) the distance from the nearest obstacle, and (d) the minimum distance from the nearest wall during a test run.

[4] S. Axler, P. Bourdon, and W. Ramey. Harmonic Function Theory. Springer-Verlag, New York, 2 edition, 2001.

[5] A. Bettini, P. Marayong, S. Lang, A. M. Okamura, and G. D. Hager. Vision Assited Control for Manipulation Using Virtual Fixtures. IEEE Transactions on Robotics and Automation, 20(6):953-966, Dec 2004.

[6] V. Duchaine and C. M. Gosselin. General Model of Human-Robot Cooperation Using a Novel Velocity Based Variable Impedance Control. In Proccedings of the 2nd Joint EuroHaptics Conference and Symposium on Haptic Interfaces for Virtual Environment and Teleoperator Systems, pages 446-451, 2007.

[7] S. Ekvall, D. Aarno, and D. Kragic. Online Task Recognition and Real-Time Adaptive Assistance for Computer-Aided Machine Control. IEEE Transactions on Robotics, 22:1029-1033, 2006.

[8] O. Khatib. Real-Time Obstacle Avoidance for Manipulators and Mobile Robots. Int. J. Robotics Research, 5(1):90-98, 1986.

[9] E. P. Klement, R. Mesiar, and E. Pap. Triangular Norms (Trends in Logic). Springer-Verlag, 2000.

[10] M. Li and A. M. Okamura. Recognition of Operator Motions for RealTime Assistance using Virtual Fixtures. In Proceedings of the 11th Symposium on Haptic Interfaces for Virtual Environments and Teleoperator Systems, pages 125-131, 2003.

[11] P. Marayong and A. Okamura. Speed-Accuracy Characteristics of Human-Machine Cooperative Manipulation using Virtual Fixtures with Variable Admittance. Human Factors: The Journal of the Human Factors and Ergonomics Society, 46(3):518-532, 2004.

[12] N. Nitzsche, U. D. Hanebeck, and G. Schmidt. Motion Compression for Telepresent Walking in Large Target Environments. Presence: Teleoperators \& Virtual Environments, 13(1):44-60, Feb. 2004.
[13] C. Passenberg, A. Peer, and M. Buss. A Survey of Environment-, Operator-, and Task-Adapted Controllers for Teleoperation Systems. Journal of Mechatronics (Spec. Iss. on Design \& Control Meth. in Telerobotics), 20(7):787-801, 2010.

[14] S. Payandeh and Z. Stanisic. On Application of Virtual Fixtures as an Aid for Telemanipulation and Training. In Proceedings of Symposium on Haptic Interfaces for Virtual Environments and Teleoperator Systems, pages 18-23, 2002.

[15] A. Pérez Arias and U. D. Hanebeck. A Novel Haptic Interface for Extended Range Telepresence: Control and Evaluation. In Proceedings of the 6th International Conference on Informatics in Control, Automation and Robotics (ICINCO 2009), pages 222-227, Milan, Italy, July 2009.

[16] A. Pérez Arias and U. D. Hanebeck. Wide-Area Haptic Guidance: Taking the User by the Hand. In Proceedings of the 2010 IEEE/RSJ International Conference on Intelligent Robots and Systems (IROS 2010), Taipei, Taiwan, Oct. 2010.

[17] B. Peterson, M. Wells, T. A. Furness III, and E. Hunt. The Effects of the Interface on Navigation in Virtual Environments. In Proceedings of Human Factors and Ergonomics Society Annual Meeting, pages 1496-1505, 1998.

[18] Z. Pezzementi, A. M. Okamura, and G. D. Hager. Dynamic Guidance with Pseudoadmittance Virtual Fixtures. In Proceedings of the International Conference on Robotics and Automation, 2007.

[19] R. Volpe. Techniques for Collision Prevention, Impact Stability, and Force Control by Space Manipulators. Teleoperation and Robotics in Space, pages 175-208, 1994. 